\title{
Levantamento georreferenciado de fisioterapeutas no Brasil
}

\author{
Georeferenced survey of physiotherapists in Brazil \\ Encuesta georreferenciada de fisioterapeutas en Brasil
}

\section{Resumo}

A disponibilidade de profissionais é um fator desafiador para gestão dos recursos em saúde, sobretudo no Brasil, um país com processo de interiorização e urbanização diferenciado entre as regiões. A distribuição de fisioterapeutas brasileiros ainda é pouco estudada. Georreferenciamento é a conversão da descrição de um local em uma posição na superfície da Terra e uma ferramenta útil na localização de recursos humanos em saúde. Assim, objetivou-se analisar a distribuição de fisioterapeutas no território brasileiro por meio do mapeamento desses profissionais nos Conselhos Regionais de Fisioterapia e Terapia Ocupacional e unidades federativas. Estudo documental descritivo, número de fisioterapeutas por Conselho Regional obtido via consulta aos portais eletrônicos oficiais ou solicitação dos dados via e-mail dos Conselhos. Foi calculado o número de fisioterapeutas por 10 mil habitantes, ou seja, a proporção da distribuição de fisioterapeutas de acordo com os estados e também para cada Conselho Regional. Para a construção do mapa, foi utilizado o programa GeoDa versão 1.18.0. Houve aumento no número de profissionais em relação a estudos anteriores, com predominância de profissionais na região Sudeste, Nordeste e Sul. Essas disparidades podem ser associadas ao nível de urbanização, população total e taxa de envelhecimento regional.

Palavras-chave: Análise espacial; Mapeamento geográfico; Fisioterapeutas.

\begin{abstract}
The availability of professionals is a challenging factor for the management of health resources, especially in Brazil, a country with a process of interiorization and urbanization that differs between regions. The distribution of Brazilian physiotherapists is still poorly studied. Georeferencing is the conversion of the description of a location to a position on the Earth's surface and is a useful tool in locating human resources in health. Thus, the objective was to analyze the distribution of physiotherapists in the Brazilian territory through the mapping of these professionals in the Regional Councils of Physiotherapy and Occupational Therapy and federative units. Descriptive documental study, number of physical therapists per Regional Council established by consulting official electronic portals or requesting data by email from the Councils. The number of physiotherapists per 10,000 inhabitants was calculated, that is, a proportion of the distribution of physiotherapists according to the states and also for each Regional Council. For the construction of the map, it was used in GeoDa version 1.18.0. There was an increase in the number of professionals compared to previous studies, with a predominance of professionals in the Southeast, Northeast and South. These disparities can be associated with the level of urbanization, total population and regional aging rate.
\end{abstract}

Keywords: Spatial analysis; Geographic mapping; Physical Therapists.

\section{Resumen}

La disponibilidad de profesionales es un factor desafiante para la gestión de los recursos de salud, especialmente en Brasil, un país con un proceso de interiorización y urbanización que difiere entre regiones. La distribución de los fisioterapeutas brasileños aún está poco estudiada. La georreferenciación es la conversión de la descripción de una ubicación a una posición en la superficie de la Tierra y es una herramienta útil para localizar recursos humanos en salud. Así, el objetivo fue analizar la distribución de fisioterapeutas en el territorio brasileño a través del mapeo de estos profesionales en los Consejos Regionales de Fisioterapia y Terapia Ocupacional y unidades federativas. Estudio documental descriptivo, número de fisioterapeutas por Consejo Regional obtenido consultando portales electrónicos oficiales o solicitando datos vía correo electrónico a los Consejos. Se calculó el número de fisioterapeutas por cada 10.000 habitantes, es decir, la proporción de la distribución de fisioterapeutas según los estados y también por cada Consejo Regional. Para la construcción del mapa se utilizó el programa GeoDa versión 1.18.0. Hubo un incremento 
en el número de profesionales respecto a estudios previos, con predominio de profesionales en el Sureste, Nordeste y Sur, disparidades que pueden estar asociadas al nivel de urbanización, población total y tasa de envejecimiento regional.

Palabras clave: Análisis espacial; Mapeo geográfico; Fisioterapeutas.

\section{Introdução}

A disponibilidade de profissionais é um fator desafiador para gestão dos recursos em saúde, sobretudo no Brasil, um país de dimensões continentais e com um processo de interiorização e urbanização diferenciado entre as regiões. A desigualdade espacial observada na distribuição desses profissionais é evidenciada quando observadas unidades da federação com maior renda domiciliar média per capita e as capitais de estado, o que revela um desequilíbrio na estrutura do sistema nacional de saúde e da capacidade de atender adequadamente à população (dos Santos, Manzano \& Krein,2021).

Fisioterapeutas são profissionais que atuam na reabilitação e promoção da saúde em todos os seus níveis, sendo fundamentais desde a prevenção e redução de riscos relacionados à saúde até a recuperação funcional e melhora da qualidade de vida. Seu trabalho engloba a avaliação, manutenção e restauração da biomecânica (Sampaio et al., 2019).

No Brasil, os órgãos responsáveis por fiscalizar a atuação dos fisioterapeutas e registrar o quantitativo desses profissionais são o Conselho Federal de Fisioterapia e Terapia Ocupacional (COFFITO) e os Conselhos Regionais de Fisioterapia e Terapia Ocupacional (CREFITO). Segundo a Lei n 6.316 de 1975, os Conselhos Regionais devem "publicar, anualmente, seu orçamento e respectivos créditos adicionais, os balanços, a execução orçamentária, o relatório de suas atividades e a relação dos profissionais registrados" (Lei n $\left.{ }^{\circ} 6.316,1975\right)$.

O georreferenciamento, primeiro processo de análise de dados espaciais, é a conversão da descrição de um local em uma posição na superfície da Terra. Atualmente é uma ferramenta que pode ser acessada por variados instrumentos e softwares, sendo os principais: mapas cadastrais (padrão-ouro), o Sistema de Posicionamento Global (GPS) e o Google Earth (Ribeiro, Olhero, Teixeira, Magalhães \& Pina, 2014).

A correlação espacial é muito significativa nos estudos em Saúde e data desde 1789, quando casos de febre amarela em Nova York foram georreferenciados. Desde então essa técnica tem sido utilizada para o mapeamento de diversas doenças, estudos epidemiológicos e análises de distribuição de serviços e recursos humanos (Ribeiro, Olhero, Teixeira, Magalhães \& Pina, 2014).

No Brasil, começaram a ser realizadas análises estatísticas em Saúde a partir de 1931 e em 1975 foi instituída a Pesquisa de Assistência Médico-Sanitária (AMS) pelo Instituto Brasileiro de Geografia e Estatística (IBGE). Com o avanço das tecnologias para essa análise, houve alterações na coleta e análise de dados e a incorporação da localização geográfica do estabelecimento de atuação desses profissionais, por meio do Global Positioning System - GPS, foi um diferencial da edição de 2009 da pesquisa de AMS (Instituto Brasileiro de Geografia e Estatística, 2009)

Atualmente, o uso das ferramentas de análise espacial nos estudos em Saúde tem aumentado e se mostrado vantajoso na esfera de saúde pública, uma vez que são uma tecnologia eficiente ao facilitar processos de informação e tomada de decisão (Rebolledo, Chiaravalloti Neto \& Giatti, 2018). Em vista disso, a investigação da distribuição dos profissionais médicos é bastante estudada. No entanto, quando observada especificamente a distribuição de fisioterapeutas no território brasileiro essa análise ainda é pouco documentada. Considerando o papel desse profissional nos mais diversos setores e níveis de atenção, analisar sua distribuição é uma maneira de direcionar a melhor gestão desse grupo e garantir o acesso aos seus serviços (Gonzalo, \& Malik, 2018).

Dessa maneira, a investigação da distribuição dos fisioterapeutas no território brasileiro é uma importante ferramenta no gerenciamento do acesso da população a esses serviços e o georreferenciamento é uma alternativa atualizada e de baixo 
custo. O objetivo deste estudo foi analisar a distribuição de fisioterapeutas no território brasileiro por meio do mapeamento desses profissionais em seus respectivos Conselhos Regionais de Fisioterapia e Terapia Ocupacional (CREFITO) e unidades federativas (UF).

\section{Metodologia}

Foi realizada uma pesquisa documental, assim definida por tomar como fonte de coleta de dados apenas documentos, ou seja, fontes primárias como leis, ofícios e relatórios oficiais. Também enquadra-se na modalidade descritiva, uma vez possui a função principal de descrever de modo exato as características quantitativas de uma população (Marconi \& Lakatos, 2021). Assim, o número de fisioterapeutas por CREFITO foi obtido via consulta aos portais eletrônicos dos Conselhos. Todos os 18 CREFITOs foram considerados nessa análise e para aqueles nos quais não foi possível identificar os dados necessários nos portais foi feita a solicitação via e-mail. Os dados foram coletados até o mês de dezembro de 2020 e revelaram um total de 278.708 fisioterapeutas no território brasileiro.

Quanto ao número de habitantes por UF, foi considerada a estimativa para o ano de 2020 do Portal Cidades do Instituto Brasileiro de Geografia e Estatística (IBGE) (https://cidades.ibge.gov.br/). Por ser um estudo documental foi dispensada a apreciação da Comissão Nacional de Ética em Pesquisa. Por meio de análise estatística descritiva, foi calculado o número de fisioterapeutas por 10 mil habitantes, ou seja, a proporção da distribuição de fisioterapeutas de acordo com os estados e também para cada CREFITO.

Para a construção do mapa, foi utilizado o programa de georreferenciamento GeoDa versão 1.18.0. A base cartográfica utilizada foi obtida no portal do IBGE com escala de 1: 250.000. Primeiramente foi construído um mapa de densidade populacional do número de fisioterapeutas em cada estado. Em seguida, construiu-se um mapa de tendências número de fisioterapeutas por 10 mil habitantes.

\section{Resultados}

Quanto ao número de fisioterapeutas por estado e região (Tabela 1), foi encontrada predominância de profissionais nas regiões sudeste e nordeste. Em contrapartida, a região norte apresentou o menor número de profissionais.

Tabela 1. Número de fisioterapeutas por região e unidade federativa (UF) e população estimada em 2020.

\begin{tabular}{cccc}
\hline Região & UF & População Estimada 2020 & $\mathbf{N}^{\mathbf{0}}$ fisioterapeutas por estado \\
\hline GO & DF & 7.113 .540 & 7.645 \\
Centro-oeste & MT & 3.055 .149 & 3.531 \\
& MS & 3.526 .220 & 3.431 \\
& Total parcial: & 2.809 .394 & 20.559 \\
Nordeste & BA & 16.504 .303 & 17.163
\end{tabular}


Research, Society and Development, v. 10, n. 14, e536101422392, 2021

(CC BY 4.0) | ISSN 2525-3409 | DOI: http://dx.doi.org/10.33448/rsd-v10i14.22392

\begin{tabular}{|c|c|c|c|}
\hline & & & \\
\hline & $\mathrm{PE}$ & 9.616 .621 & 9.497 \\
\hline & PB & 4.039.277 & 5.974 \\
\hline & MA & 7.114 .598 & 3.992 \\
\hline & PI & 3.281 .480 & 3.896 \\
\hline & $\mathrm{RN}$ & 3.534 .165 & 3.820 \\
\hline & $\mathrm{AL}$ & 3.351 .543 & 3.250 \\
\hline & SE & 2.318 .822 & 2.279 \\
\hline \multirow{9}{*}{ Norte } & Total parcial: & 57.374 .243 & 59.481 \\
\hline & PA & 8.690 .745 & 3.939 \\
\hline & $\mathrm{AM}$ & 4.207 .714 & 3.185 \\
\hline & RO & 1.796 .460 & 1.753 \\
\hline & TO & 1.590 .248 & 1.362 \\
\hline & AP & 861.773 & 892 \\
\hline & $\mathrm{AC}$ & 894.470 & 792 \\
\hline & $\mathrm{RR}$ & 631.181 & 626 \\
\hline & Total parcial: & 18.672 .591 & 12.549 \\
\hline \multirow{4}{*}{ Sudeste } & SP & 46.289 .333 & 79.910 \\
\hline & $\mathrm{RJ}$ & 17.366 .189 & 34.760 \\
\hline & MG & 21.292 .666 & 27.602 \\
\hline & ES & 4.064 .052 & 4.256 \\
\hline \multirow{5}{*}{ Sul } & Total parcial: & 89.012 .240 & 146.528 \\
\hline & $\mathrm{RS}$ & 11.422 .973 & 15.385 \\
\hline & PR & 11.516 .840 & 14.547 \\
\hline & SC & 7.252 .502 & 9.659 \\
\hline & Total parcial: & 30.192 .315 & 39.591 \\
\hline otal Final & & 211.755.692 & 278.708 \\
\hline
\end{tabular}

Legenda: GO: Goiás. DF: Distrito Federal. MT: Mato Grosso. MS: Mato Grosso do Sul. BA: Bahia. CE: Ceará. PE: Pernambuco. PB: Paraíba. MA: Maranhão. PI: Piauí. RN: Rio Grande do Norte. AL: Alagoas. SE: Sergipe. PA: Pará. AM: Amazonas. RO: Rondônia.TO: Tocantins. AP: Amapá. AC: Acre. RR: Roraima. SP: São Paulo. RJ: Rio de Janeiro. MG: Minas Gerais. ES: Espírito Santo. RS: Rio Grande do Sul. PR: Paraná. SC: Santa Catarina. Fonte: Autores (2021) 
Research, Society and Development, v. 10, n. 14, e536101422392, 2021

(CC BY 4.0) | ISSN 2525-3409 | DOI: http://dx.doi.org/10.33448/rsd-v10i14.22392

Quando considerada a população total de cada UF, o número de fisioterapeutas por 10 mil habitantes apresentou média de 11,35. Os estados que apresentaram valores inferiores ou iguais à média $(\mathrm{n}=16)$ foram: Pará $(4,53)$, Maranhão $(5,61)$, Amazonas (7,57), Tocantins (8,56), Acre $(8,85)$, Alagoas $(9,70)$, Rondônia $(9,76)$, Sergipe $(9,83)$, Pernambuco $(9,88)$, Roraima $(9,92)$, Amapá (10,35), Ceará (10,46), Espírito Santo (10,47), Goiás (10,75), Rio Grande do Norte (10,81), Mato Grosso $(11,20)$. Quanto às UFs que apresentaram valores superiores à média $(n=11)$ encontram-se: Bahia $(11,50)$, Piauí $(11,87)$, Mato Grosso do Sul (12,22), Paraná (12,63), Minas Gerais (12,96), Santa Catarina (13,32), Rio Grande do Sul (13,47), Paraíba $(14,79)$, São Paulo $(17,26)$, Distrito Federal $(18,10)$ e Rio de Janeiro $(20,02)$. Essa variação do número de profissionais entre os estados pode ser percebida visualmente no mapa de densidade populacional (Figura 1).

Consequentemente, o CREFITO 1 (Pernambuco, Paraíba, Alagoas, Rio Grande do Norte), CREFITO 2 (Rio de Janeiro) e CREFITO 3 (São Paulo) apresentam o maior número de fisioterapeutas por 10 mil habitantes conforme percebido no mapa de tendências (Figura 2). O CREFITO 11 (Goiás e Distrito Federal) também apresenta um valor acima da média como também evidenciado no segundo mapa. Predominantemente, estados da região norte apresentaram valores abaixo da média nacional, correspondentes aos CREFITOS 12 e 18.

Figura 1. Mapa de densidade populacional do número de fisioterapeutas por 10 mil habitantes.

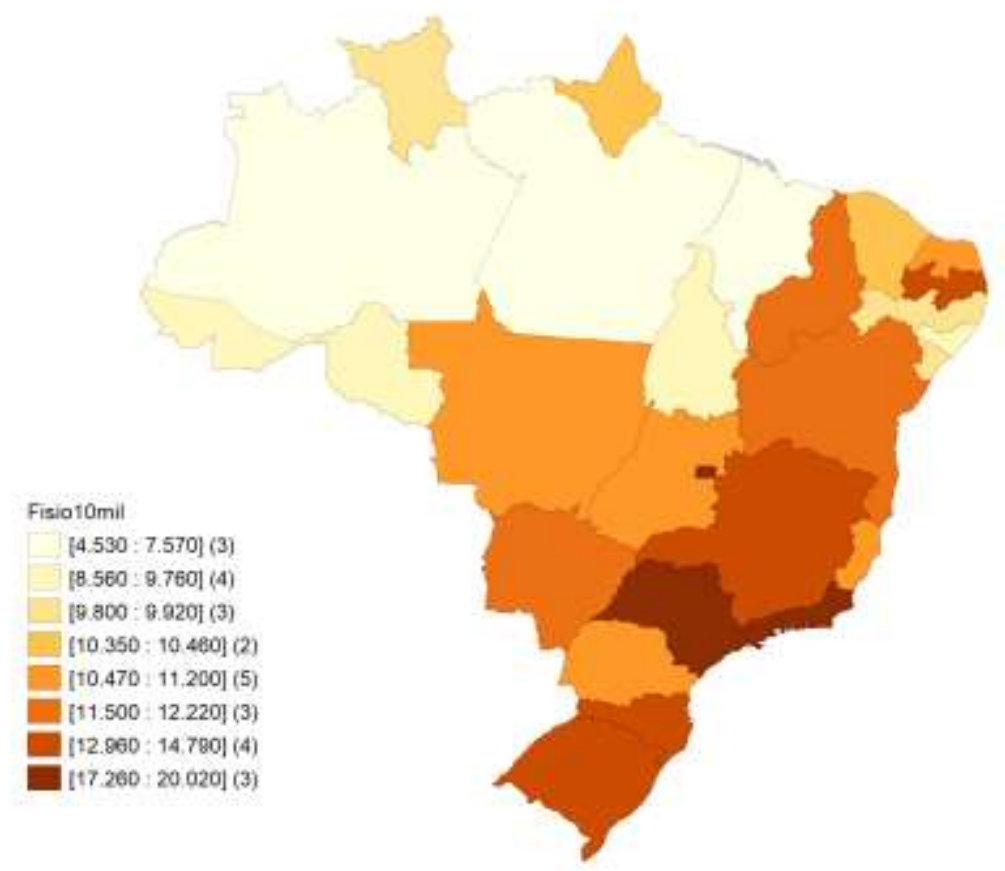

Fonte: Autores (2021).

Comentário dos autores: Por tratar-se de um mapa de densidade a coloração reflete a concentração dos profissionais. Tons mais claros revelam uma menor densidade de fisioterapeutas, enquanto as cores mais escuras revelam uma maior concentração de profissionais. Assim, fica evidente a maior densidade de profissionais nas regiões sul e sudeste. 
Figura 2. Mapa de tendências da relação do número de fisioterapeutas por 10 mil habitantes em seus respectivos CREFITOs.

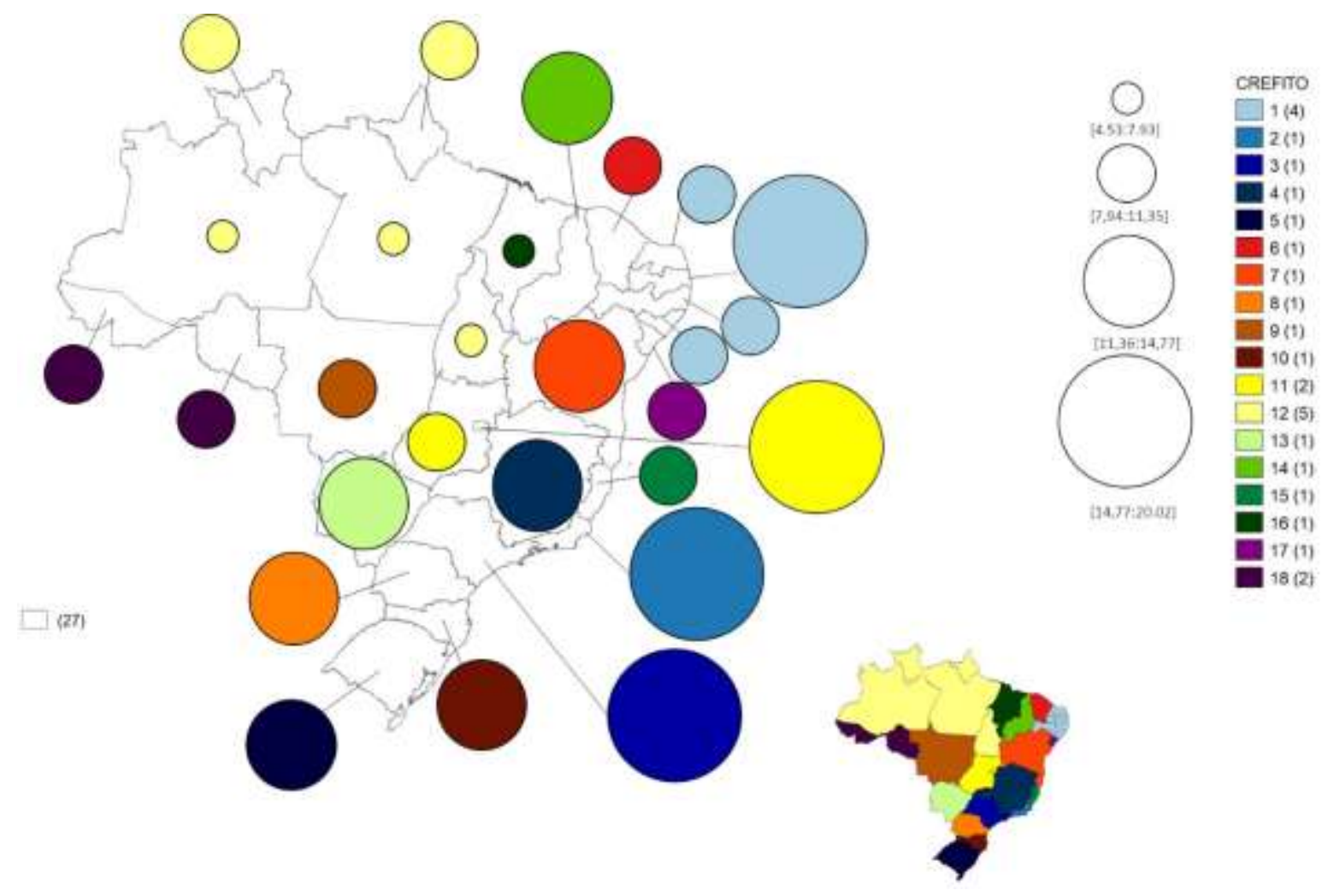

Fonte: Autores (2021).

Comentário dos autores: Por se tratar de um mapa de tendências o tamanho da circunferência indica o intervalo correspondente à quantidade de fisioterapeutas por 10 mil habitantes naquele estado. A cor da circunferência indica o CREFITO ao qual o estado pertence, conforme indicado na legenda.

\section{Discussão}

Ao utilizar o georreferenciamento foi possível analisar o panorama geral e quantificar a relação profissionalpopulação e profissional-região no território brasileiro. O número por estado é uma medida simples que torna a visualização desses dados mais individualizada e as discrepâncias ainda mais evidentes.

Em 2012, dados fornecidos pela World Confederation for Physical Therapy revelaram uma média global de 0,002 fisioterapeutas por 1000 habitantes a 2,82 por 1000 habitantes entre 69 de seus 106 países membros (Sykes., Bury, \& Myers, 2014). No presente estudo, todos os estados brasileiros encontraram uma proporção de profissionais dentro desse valor, sendo a média geral de 1,13 fisioterapeutas por 1000 habitantes.

Os resultados encontrados revelam um crescimento de $35,18 \%$ no número de fisioterapeutas no país em relação ao estudo anterior de Matsumura et al (2018), o qual apresentou um número total de 206.170 cadastrados até setembro de 2016. Isso pode ser explicado pelo crescimento de áreas dentro da Fisioterapia que já existiam, como no caso da Fisioterapia Respiratória durante a pandemia de Sars-Cov-2, e o surgimento de novos campos de atuação, como na atenção primária, as quais expandiram o repertório do fisioterapeuta. Isso contribuiu para a disseminação e reconhecimento da Fisioterapia e refletiu em um mercado de trabalho com maior demanda e possibilidades (Sampaio et al., 2019).

Outra diferença encontrada foi quanto à criação dos CREFITOs 17 e 18. Inicialmente foram instituídos 16 
Research, Society and Development, v. 10, n. 14, e536101422392, 2021

(CC BY 4.0) | ISSN 2525-3409 | DOI: http://dx.doi.org/10.33448/rsd-v10i14.22392

CREFITOs, porém, em 2017, por meio da resolução nº 484, de 13 de julho de 2017, ocorreu o desmembramento do CREFITO 7, resultando na criação do CREFITO 17 (Resolução $\mathrm{n}^{\circ}$ 484, 2017). O CREFITO 18, por sua vez, surgiu da resolução COFFITO n 490, de 18 de dezembro de 2017 (Resolução $n^{\circ}$ 490, 2017). Esse desmembramento também é resultado do aumento de profissionais na região Norte e Centro-Oeste, uma vez que, devido ao maior número, novas reivindicações e demandas tiveram que ser atendidas de forma mais regionalizada.

Dois fatores estruturais têm sido investigados como responsáveis pelo aumento da demanda por profissionais de saúde: a urbanização e o envelhecimento populacional. Os centros urbanos apresentam condições que favorecem a fixação dos profissionais além de ser o local onde se encontram os centros de formação e aperfeiçoamento. O estudo de Sampaio et al. (2019) revela que a maior concentração de fisioterapeutas, em áreas mais povoadas, pode ser associada ao tamanho da população e à viabilidade de acesso aos serviços de saúde, igualmente observado em outras profissões. Isso interfere, por sua vez, na dificuldade do acesso a serviços de fisioterapia em áreas rurais que podem experimentar escassez de atendimento. Quanto ao envelhecer, quanto mais avançada é a idade, maiores são as chances de procura de serviços de saúde. O estudo de Cruz, Vieira, Carneiro, Costa, e Caldeira (2020), aponta barreiras geográficas como um dos fatores que impediam o acesso de idosos a atendimentos, sobretudo para aqueles que apresentavam condição de vulnerabilidade socioeconômica, o que faz com que cada vez mais esses centros de atendimento se desloquem para áreas de mais fácil acesso.

Nesse sentido, no presente estudo observou-se concordância com os achados de Matsumura et al.(2018) os quais evidenciaram uma menor proporção de profissionais na região Norte e Centro-Oeste.

No Brasil, a irregularidade da distribuição de profissionais de saúde tem sido atribuída ao processo de interiorização heterogênea, com causalidade histórica e econômica. Portanto, a disseminação da fisioterapia e formação de fisioterapeutas também é influenciada por esses fatores (Bispo Júnior, 2009). Foi em São Paulo que a Fisioterapia começou a ser difundida pelo Brasil, por meio do Hospital das Clínicas da Universidade de São Paulo (Marques \& Sanches, 1994). No entanto, apenas em 1969 a Fisioterapia foi regulamentada e reconhecida como curso superior (Brandenburg, Barbosa \& Martins, 2012). Desde então, os profissionais buscam expandir sua atuação e reconhecimento, flexibilizando o monopólio de outras áreas, principalmente no Sistema Único de Saúde (SUS). Dessa maneira, o fato de ser uma profissão relativamente recente e que vem conquistando o mercado contribui para a irregularidade da distribuição dos profissionais.

O maior número de fisioterapeutas na região sudeste, nordeste e sul mostraram concordância com os estudos de Tavares, Costa, Oishi e Driusso (2016), o qual encontrou que nessas regiões o maior número de metrópoles e municípios de grande porte se constituíram como os principais locais de atuação de fisioterapeutas. A razão de fisioterapeutas por 1.000 habitantes nesse estudo variou entre 0,07 (Municípios de pequeno porte do Norte) e 0,52 (Metrópoles do Sul), indicando importante variação na oferta deste profissional.

O estudo de Dos Santos, Manzano e Krein (2021) igualmente demonstra uma prevalência de profissionais de saúde nas regiões Sudeste, Sul e no Distrito Federal ao analisar a distribuição de médicos e enfermeiros em relação à população total de cada estado, sobretudo durante a pandemia de COVID-19.

Outro fator importante para o aumento do número de fisioterapeutas é o investimento governamental, sobretudo na educação superior. As políticas públicas de educação e saúde são ferramentas que estimulam a preparação de novos profissionais em sua região. O estudo de Sampaio et al. (2019) ainda argumenta que quanto menor o número de programas de formação de terapeutas em certas regiões do Brasil menor o quantitativo de fisioterapeutas trabalhando nessas regiões, gerando uma força de trabalho insuficiente.

Além disso, a relação do número de profissionais em cada localidade é um dado necessário para informar a população da disponibilidade de serviços, de forma rápida e atualizada. O estudo de Shah, Milosavljevic, Trask e Bath (2019), que analisa 
a distribuição de fisioterapeutas no Canadá, revela que a distribuição geográfica adequada de recursos humanos e serviços de saúde é um componente-chave para garantir o acesso equitativo aos cuidados de saúde.

Em vista disso, o georreferenciamento é um instrumento que auxilia profissionais e gestores a atuarem estrategicamente e melhorarem seus serviços de saúde por meio de uma oferta suficiente de profissionais qualificados, integrando um processo de territorialização. Com a atualização dos dados simultaneamente, o uso de mapas configura não somente um conjunto de instrumentos de visualização e organização desses dados, mas um processo participativo e dinâmico, que envolve os diversos setores da sociedade, desde a coleta e sistematização de informação até a confecção. Em saúde, o mapeamento permite sobrepor os diferentes dados e a identificação de demandas a serem supridas, o que resulta em um planejamento mais efetivo (Colussi \& Pereira, 2016). Isso pode ser observado no presente estudo, uma vez que foi possível observar essas disparidades regionais e fornecer esses dados de forma objetiva e ilustrada, além de registrar o crescimento do número de fisioterapeutas nos últimos anos.

\section{Conclusão}

Por meio da correlação espacial de fisioterapeutas no território brasileiro foi possível identificar uma menor concentração de profissionais nas regiões Norte e Centro-Oeste, sendo essas regiões com o maior número de estados com quantidade de fisioterapeutas abaixo da média nacional encontrada de 11,35 por 10 mil habitantes. Em regiões economicamente mais ativas quanto a aspectos de urbanização e crescimento populacional foram encontrados os maiores valores (Sudeste, Sul e Distrito Federal).

Tornar as ferramentas de georreferenciamento mais acessíveis é uma forma, não apenas de registrar dados, mas também de parametrizar regiões e estabelecer metas para melhor suprir suas demandas de acesso aos serviços fisioterapêuticos. A partir do presente estudo percebeu-se que para tornar essa distribuição mais homogênea e o acesso à fisioterapia mais igualitário é essencial que os órgãos responsáveis busquem estratégias e acompanhem as demandas dos profissionais e dos usuários, sobretudo por parte dos Conselhos Federal e Regionais de Fisioterapia e Terapia Ocupacional, de acordo com a realidade de cada região e estado. Estudos futuros podem investigar mais a fundo essa distribuição, acrescentando a análise ao nível das cidades e do Cadastro Nacional de Estabelecimentos de Saúde.

\section{Agradecimentos}

Agradecimentos especiais aos Conselhos Regionais de Fisioterapia e Terapia Ocupacional que prontamente disponibilizaram os dados necessários para a realização desta pesquisa.

\section{Referências}

Bispo Júnior, J. P. (2009). Formação em fisioterapia no Brasil: reflexões sobre a expansão do ensino e os modelos de formação. História, Ciências, SaúdeManguinhos, 16, 655-668. 10.1590/S0104-59702009000300005

Brandenburg, C., Barbosa, A., \& Martins, T. Fisioterapia: história e educação (2012, junho). In Anais do XI encontro cearense de história da educação, Fortaleza, CE, 11.

Colussi, C. F., \& Pereira, K. G (2016). Territorialização Como Instrumento Do Planejamento. Florianópolis: Universidade Federal de Santa Catarina.

Cruz, P. K. R., Vieira, M. A., Carneiro, J. A., Costa, F. M. D., \& Caldeira, A. P. (2020). Dificuldades do acesso aos serviços de saúde entre idosos não institucionalizados: prevalência e fatores associados. Revista Brasileira de Geriatria e Gerontologia,23(6), 1-13. 10.1590/1981-22562020023.190113.

dos Santos, A. L., Manzano, M., \& Krein, A. (2021). Heterogeneidade da distribuição dos profissionais de saúde no Brasil e a pandemia Covid-19. Cadernos do Desenvolvimento, 16(28), 197-219. http://www.cadernosdodesenvolvimento.org.br/ojs-2.4.8/index.php/cdes/article/view/557.

Gonzalo, V. N., \& Malik, A. M. (2018). Gestão em saúde. Rio de Janeiro: Guanabara Koogan. 
Research, Society and Development, v. 10, n. 14, e536101422392, 2021

(CC BY 4.0) | ISSN 2525-3409 | DOI: http://dx.doi.org/10.33448/rsd-v10i14.22392

Instituto Brasileiro de Geografia e Estatística (2009). Pesquisa de assistência médico sanitária. http://www.ibge.gov.br/home/estatistica/populacao.

Lei $n^{\circ}$ 6.316, de 17 de dezembro de 1975 (1975). Cria o Conselho Federal e os Conselhos Regionais de Fisioterapia e Terapia Ocupacional e dá outras providências. Diário Oficial da União. Brasília, DF: Câmara dos deputados.

Marconi, M. A., \& Lakatos, E. M. (2021) Fundamentos de metodologia científica. São Paulo: Atlas.

Marques, A. P., \& Sanches, E. L. (1994). Origem e evolução da fisioterapia: aspectos históricos e legais. Fisioterapia e Pesquisa, 1(1), 5-10. 1590/fpusp.v1i1.75027

Matsumura, E. S. D. S., Sousa, A. S., Guedes, J. A., Teixeira, R. C., Kietzer, K. S., \& Castro, L. S. D. F. (2018). Distribuição territorial dos profissionais fisioterapeutas no Brasil. Fisioterapia e Pesquisa, 25 (3), 309-314. 10.1590/1809-2950/17027025032018.

Rebolledo, E. A. S., Chiaravalloti Neto, F., \& Giatti, L. L. (2018). Experiencias, beneficios y desafíos del uso de geoprocesamiento para el desarrollo de la atención primaria de salud. Revista Panamericana de Salud Pública, 42 (8), 1-13. 10.26633/RPSP.2018.153

Resolução $n^{\circ}$ 490, de 18 de dezembro de 2017 (2017). Promove o desmembramento da região territorial do CREFITO-9 e determina a realização de eleições diretas para preenchimento de cargos de Conselheiros Efetivos e Suplentes do Conselho Regional de Fisioterapia e Terapia Ocupacional da $18^{\text {a }}$ Região, como condição para sua definitiva instalação. Diário Oficial da União. Brasília, DF: Conselho Federal de Fisioterapia e Terapia Ocupacional (COFFITO).

Resolução $n^{\circ}$ 484, de13 de julho de 2017 (2017). Promove o desmembramento da região territorial do CREFITO-7 e determina a realização de eleições diretas para preenchimento de cargos de Conselheiros Efetivos e Suplentes do Conselho Regional de Fisioterapia e Terapia Ocupacional da $17^{a}$ Região, como condição para sua definitiva instalação. Diário Oficial da União. Brasília, DF: Conselho Federal de Fisioterapia e Terapia Ocupacional (COFFITO).

Ribeiro, A. I., Olhero, A., Teixeira, H., Magalhães, A., \& Pina, M. F. (2014). Tools for address georeferencing-limitations and opportunities every public health professional should be aware of. PLoS One, 9(12), 1-13. 10.1371/journal.pone.0114130.

Sampaio, R. F., Wan Der Maas, L., Marães, V. R., Neves, J. A., Vaz, D. V., Nóbrega, R. A., \& Ocarino, J. M. (2019). Physical Therapist Education and the Labor Market in Brazil: Advances and Challenges. Physical therapy, 99(8), 977-988. 10.1093/ptj/pzz055.

Shah, T. I., Milosavljevic, S., Trask, C., \& Bath, B. (2019). Mapping physiotherapy use in Canada in relation to physiotherapist distribution. Physiotherapy Canada, 71(3), 213-219. 10.3138/ptc-2018-002.

Sykes, C., Bury, T., \& Myers, B. (2014). Physical therapy counts: counting physical therapists worldwide. BMC Health Services Research, 14(2), 1-1. $10.1186 / 1472-6963-14-\mathrm{S} 2-\mathrm{O} 23$

Tavares, L. R. C., Costa, J. L. R., Oishi, J., \& Driusso, P. (2016). Distribuição territorial de fisioterapeutas no Brasil: análise do Cadastro Nacional de Estabelecimentos de Saúde CNES/2010. ConScientiae saúde, 15(1), 53-61. 10.5585/conssaude.v15n1.6152. 